\title{
Gobernanza de las políticas de proximidad: análisis de calidad y rendimiento democrático de los Consejos Locales Bienestar (Murcia, España)*
}

\author{
Enrique Pastor Seller**
}

\begin{abstract}
Sumario: 1. Presentación: contextualización y ruta metodológica; 2. Democratización vs. eficacia/calidad de las políticas sociales locales; 3 . El porqué de la participación; 4. Políticas de participación ciudadana en el ámbito local: referentes internacionales y nacionales; 5 . La participación en la reforma de las Leyes autonómicas de servicios sociales en España; 6. Dimensiones y mecanismos de participación en las políticas sociales municipales; 7. Rendimiento democrático de los órganos de participación institucionalizados (análisis de caso región de Murcia, España); 8. Conclusiones y oportunidades para ejercer ciudadanía.
\end{abstract}

Summary: 1. Introduction; 2. Democratization vs. effectiveness/quality of local social policies; 3.The why participation; 4. Policy of citizen participation in local, nacional and international benchmarks; 5 . Participation in the reform of social services regional laws in Spain; 6. Size and mechanisms for participation in municipal social policies; 7. Democratic performance of institucionalized participatory bodies (case study Murcia, Spain); 8. Conclusions and opportunities to exercise citizenship.

Palabras clave: participación cívica; desarrollo comunitario; servicios sociales municipales; políticas sociales municipales; intervención comunitaria.

KEY WORDs: civic participation; comunity development; local social services; local social policies; comunitary intervention.

El artículo presenta un análisis y evaluación de las oportunidades, contribuciones y limitaciones que presentan los órganos de participación institucionalizada en materia de servicios sociales municipales para profundizar en los procesos democratizadores de las políticas sociales públicas gestionadas por las administraciones locales, así

\footnotetext{
* Artículo recibido en oct. 2010 y aprobado en ene. 2011.

** Profesor titular del Departamento de Sociología y Política Social de la Facultad de Economía y Empresa de la Universidad de Murcia. Decano de trabajo social de la Universidad de Murcia. Dirección: Universidad de Murcia. Escuela de Trabajo Social. Campus de Espinardo - Espinardo — 30.071, Murcia, España. E-mail: epastor@um.es.
} 
como las propuestas y alternativas que desde sus actores participantes supondría su intensificación para la gobernanza local y la eficacia de centros, organizaciones y profesionales de la intervención social. Para su adecuada ilustración y contextualización se alude a la línea de reformas que introducen las recomendaciones y normas de carácter internacional, nacional, así como las nuevas leyes de servicios sociales autonómicas y los hallazgos y conclusiones obtenidos en una investigación empírica sobre participación ciudadana en las políticas sociales de la región de Murcia, contrastando y comparando sus resultados con realidades y tendencias observadas en otros estudios y experiencias.

Proximity social policies government: quality and democratic improvement analysis of local council of well being (Murcia, Spain)

Te paper presents an analysis and an evaluation of the oprtunities, contributions and limitations that the institutionalizated participation structures present in the field of local social services to study in depth the democratic process in local public policies, as well as the proposals and alternatives that, from theis participants would suposse to intensify for the local governship and for the , centers efficacy, organizations and social intervention professions. For its adecuated illustration and contextualization we mention the reform lines that introduce the new autonomic social services laws and also the findings and conclusions obtained trough an empirical reserche about citizenship in The region de Murcia social policies contrasting and comparing their results with realities and trends observed in others studies and experiencies.

\section{Presentación: contextualización y ruta metodológica}

El artículo presenta un análisis y evaluación de las oportunidades y limitaciones de la participación institucionalizada en las políticas sociales municipales, comparando hallazgos con realidades y tendencias observadas en otros estudios y experiencias de ámbito nacional e internacional y, más concretamente, las contribuciones, limitaciones y tendencias más significativas de los consejos territoriales locales en su capacidad para influir en los procesos de democratización y eficacia de las políticas sociales en el ámbito local.

Centrar la investigación en la participación ciudadana, en el ámbito de los servicios sociales municipales y a través de instrumentos participativos concretos y regulados, como son los consejos locales ${ }^{1}$ permite delimitar un fenómeno emergente y complejo; adaptarse a la literatura especializada entorno a la democracia asociativa; disponer de un marco institucionalizado y una

\footnotetext{
${ }^{1}$ Órganos de participación recogidos en todas las leyes autonómicas de servicios sociales.
} 
lógica normativa concreta y consolidada a la que referirse y establecer análisis comparativos entre contextos, mecanismos y prácticas. El estudio, cuyos resultados a continuación se presentan, ha permitido disponer de una evaluación de las formas de organización de la participación ciudadana en los servicios sociales de cada una de las corporaciones locales de la región, elaborar tipologías y análisis comparativo de casos, así como evaluar el impacto y contribución de los consejos en la profundización democrática de las políticas públicas locales en materia de servicios sociales y la mejora en la eficacia de la gestión de los servicios y prestaciones sociales de atención primaria.

El enfoque metodológico empleado se ha basado en la utilización de técnicas, fundamentalmente de carácter cualitativo, lo que ha permitido recoger los discursos, las opiniones, voces, sugerencias e interpretaciones de los protagonistas en los procesos de participación ciudadana en materia de servicios sociales de ámbito local. El estudio de casos ha permitido la proximidad y participación de los actores que construyen la realidad, superando, de esta forma, meras intenciones descriptivas o contables para explicar los motivos que conducen a una realidad determinada. A su vez, este método presenta una importante virtud, como es la de producir conocimientos relevantes a partir de la combinación de técnicas y desde un contexto participativo de los sujetos/actores. Considerando la complejidad del fenómeno a conocer, se han empleado diversas técnicas para la recogida de datos con el fin de incrementar la eficiencia de las mismas y solucionar el problema que representaba el análisis de la complejidad causal, fundamentalmente, la entrevista a responsables/técnicos de todas las corporaciones locales de la región, entrevistas en profundidad a los actores participantes y grupos de discusión con participantes estratégicos. Atendiendo a la máxima representatividad de los discursos existentes, se contempló la diversidad de los actores participantes en los mecanismos de participación objeto de estudio, concretamente los agentes: políticos (tanto del gobierno como de los diferentes partidos políticos de la oposición), técnicos (relevancia, heterogeneidad, responsabilidad e implicación directa) y asociativos (cualificación y contraste de discursos, diversidad, máxima representatividad y permanencia como vocal en el mecanismo participativo). Para su contraste y ampliación se emplearon la recopilación, síntesis y análisis documental (teórico, normativo y empírico) en una doble dirección: interna y externa a las unidades de observación. Todo ello ha permitido el contraste de los datos obtenidos en los cuestionarios, las entrevistas y los grupos de discusión con aquellos derivados del análisis documental y de contenido; desde una aproximación comprensiva y reflexiva por parte de los actores de la participación ciudadana en la gestión de la política de los servicios sociales municipales. 


\section{Democratización vs. eficacia/calidad de las políticas sociales locales}

En la actualidad emerge una progresiva demanda por la democracia directa y la participación ciudadana en la gestión pública, convirtiéndose en un asunto central y de actualidad en el debate social y académico y con un significado sustantivo en los políticos y profesionales vinculados (directa o indirectamente) con las políticas sociales. La complejidad e interdependencia de los fenómenos y hechos sociales, así como las situaciones de dificultad que atraviesan personas, familias, grupos y comunidades requiere de compromisos, competencias e interacciones de los diferentes actores sociales (públicos y cívicos), convirtiéndose la participación en un elemento consustancial en este nuevo contexto pluralista y relacional. Pero apostar por la participación supone iniciar un camino "pedregoso" que requiere resolver, de manera dinámica, los problemas que afectan a las capacidades y oportunidades de representación, gobernabilidad y legitimidad en las democracias actuales y adoptar decisiones respecto a la forma y sentido de materializar el principio de autonomía/autodeterminación y, por consiguiente, de ciudadanía (activa vs. subsidiaria) en los contextos microsociales.

La democracia se alimenta del capital de confianza de las personas en el futuro y en su propia capacidad, real y percibida, de acción e influencia para la transformación socio-política. La crisis de confianza de los ciudadanos que se constata en la actualidad en los diferentes estudios de opinión pública, especialmente ante la situación de evidente recesión económica y crisis social, augura malos tiempos para la democracia y la construcción negociada e integradora de las políticas sociales. A pesar de ello, debemos adoptar una actitud y compromiso positivo respecto del cambio social y la superación de las dificultades desde las capacidades humanas y mediante la profundización democrática, entendida como ampliación, en términos cuantitativos y cualitativos, tanto de los actores (número y pluralidad representativa) que pueden intervenir en los procesos participativos, como de las cuestiones y los niveles de influencia y producción de políticas sociales por parte de los diferentes actores implicados (políticos, técnicos y ciudadanos). La participación aporta sustantivos beneficios a la dinámica organizacional y comunitaria al proporcionar una progresiva adecuación del funcionamiento de las instituciones, romper la apatía y desconfianza ciudadana, ofrecer a los representantes herramientas para evaluar y mejorar la gestión de los asuntos públicos, permitir a la ciudadanía reconquistar y recuperar el espacio público, generar capital social, potenciar sentimientos comunitarios; permitir que la "política se socialice" y reforzar las 
decisiones a adoptar o, incluso, adoptadas. De ahí que la participación se haya convertido en un asunto de interés preferente y transversal en la agenda de los gobiernos y profesionales que desean implementar una gestión política y/o técnica eficaz orientada a mejorar el bienestar social y la calidad de vida de la ciudadanía y de los usuarios de centros/servicios. ${ }^{2}$ Pero esta centralidad requiere de instrumentos que permitan una adecuada y transparente canalización de la misma y de procesos de decisión y gestión descentralizada, colegiada, ágil y co-responsable en los asuntos públicos sociales municipales.

Las novedades y debates en torno al municipalismo se concretan en el tránsito desde el tradicional gobierno local hasta la actual "governance" local - gobierno en red o gobierno relacional - Los gobiernos locales ya no dialogan con una comunidad homogénea, sino con múltiples comunidades e identidades que conviven y/o se interrelacionan en, desde y con el territorio. Los ciudadanos no nos conformamos con exigir la calidad y eficacia en la prestación de los servicios públicos; queremos, también, participar en la misma definición y articulación del desarrollo local a escala humana. Ello solo será posible con el concurso de los ciudadanos, grupos y entidades organizados; lo que requiere reconocer, conciliar y reconciliar intereses, tender puentes, equilibrar preferencias y construir compromisos colectivos de gobernabilidad. En este sentido, en coincidencia con Navarro (2008), la extensión y diversificación en la oferta de participación produce cierto efecto movilizador en el sentido de que los ciudadanos con similares orientaciones hacia la democracia local y/u organizacional, se implican más cuando existen más oportunidades, cuando viven en un contexto participativo, proporcionando así un aumento del ejercicio de ciudadanía activa. De ahí que el reto sea crear condiciones y espacios de participación/implicación ciudadana que generen oportunidades reales y favorables para la deliberación y construcción colectiva de las políticas sociales a partir de la conformación de preferencias sólidas e informadas entre la ciudadanía en el complejo universo relacional.

El gobierno local se nos presenta como un escenario privilegiado de participación, siendo especialmente visibles la emergencia de los espacios/mecanismos participativos en éste ámbito. Se considera que la escala de gobierno menor facilita el desarrollo de instrumentos participativos, en tanto permite el diálogo y la interacción personal e interinstitucional. La proximidad de los te-

\footnotetext{
${ }^{2}$ La participación, en coincidencia con Subirats, en la elaboración de las políticas sociales es "una herramienta fundamental para mejorar el diseño, evaluar los servicios y detectar las necesidades reales de la población" (Subirats, 2007:57).
} 
mas a los ciudadanos provoca una mayor disposición por su parte a implicarse y participar; por lo que es necesario innovar en mecanismos que permiten escuchar las voces de la ciudadanía en los procesos de toma de decisiones a nivel local, más allá de proveer de información al ciudadano sobre la actividad de la administración municipal (unidireccional), por otra parte requisito previo, sino incidiendo en flujos multidireccionales en red, es decir en la vinculación e influencia ciudadana en la toma de decisiones de su gobierno local. La mayor parte de los gobiernos locales europeos se hallan, como señalan diferentes investigaciones y autores ${ }^{3}$ desde hace al menos dos decenios, inmersos en procesos de reforma. Los objetivos que se han perseguido con estas reformas pueden sintetizarse en dos; por una parte, las administrativas orientadas a lograr la eficiencia, la eficacia y la calidad de las estructuras administrativas locales y sus resultados en su relación con los deseos ciudadanos y, por otra, las políticas pretenden lograr el enriquecimiento de la democracia local, normalmente en forma de mayor inclusividad y acceso de la ciudadanía a las decisiones públicas que les afectan.

\section{El porqué de la participación}

La participación ciudadana puede contener diferentes significaciones, atendiendo a los múltiples objetivos a los que puede dirigirse un proceso participativo y, en razón de estos, diseñar los mecanismos e indicadores que pueden facilitarlo y evaluarlo, respectivamente. La clasificación de los objetivos - del porqué - de la participación puede realizarse en función de diferentes criterios, destacando dos: papel otorgado a la ciudadanía y resultados que los gobernantes/responsables esperan obtener.

Respecto al criterio de poder otorgado (niveles y procesos), la literatura especializada nos refiere a las conocidas escaleras de la participación (Arnstein, 1969), donde en cada travesaño se sitúan los diversos mecanismos de participación en función del papel que otorga a la ciudadanía y a sus organizaciones en las diferentes fases de producción de las políticas públicas. En la parte más baja se sitúan los instrumentos participativos que pretenden únicamente ofrecer información. En el nivel medio aparecen diversos mecanismos que quieren dar voz, es decir dirigidos a obtener mediante la consulta la opi-

\footnotetext{
${ }^{3}$ Entre otros: Del Pino y Colino, 2003; Löffler, 2005; Brugué y Vallés, 2005; VVAA, 2005; Arriba, Calzada y Del Pino, 2006; Inap, 2008 etc.
} 
nión de los ciudadanos o de sus organizaciones. Finalmente, en la parte más alta de esta escalera metafórica aparece la participación más potente, es decir aquella que permite trasladar el poder y, por tanto, la capacidad para tomar decisiones a la sociedad civil. Estos tres objetivos se resumen como el derecho a ser informado, consultado y/o a tomar parte.

Informar y formar de manera transparente y entendible. Es habitual confundir participación con dar información; sin embargo, ésta es sólo el primer nivel o requisito previo (perspectiva del "continuum"), dado que disponer de información es imprescindible para que pueda existir participación, pero no es suficiente. La información circula en dos sentidos: ascendente, desde los ciudadanos hacia la autoridad, y descendente, desde los responsables públicos a los ciudadanos. Lo importante no es solo la cantidad de información, sino el momento (antes o depuse de haber adoptado las decisiones) y la calidad (relevancia para sus intereses y forma de ofrecerla) de ésta.

Consultar eficazmente dando evidencias de escuchar. Implica que la población no sólo conoce propuestas y decisiones, sino que tiene opciones de expresar opiniones, sugerencias y alternativas. Se produce cuando las autoridades/responsables deciden mejorar sus decisiones teniendo en cuenta la opinión de determinados grupos o individuos afectados (usuarios/as) o del público en general. Requiere que los temas planteados se encuentren en proceso de diseño o bien puedan ser potencialmente modificados, lo que implica una clara voluntad política y/o técnica en incorporar dichas opiniones y la capacidad institucional y normativa para introducir dichos cambios. La calidad de los datos obtenidos en la consulta depende en gran parte de la información que disponga el ciudadano para emitir su juicio u opinión acerca del tema consultado. El más alto nivel de consulta deliberativa es aquel basado en la interacción constructivista, o transacción, lo que supone intercambios circulares, en los que la persona, grupo y entorno se moldean recíprocamente en continuos e indeterminados procesos de influencia mutua.

Delegar responsabilidades y co-producir bienestar. Supone el tercer grado en la escalera e implica la intervención ciudadana, usuarios/as y/o representantes en el proceso de elaboración de las decisiones mediante estructuras establecidas, bien territoriales, sectoriales u organizacionales. Los participantes o usuarios, mediante acciones directas o a través de organizaciones, hacen efectivo su poder y capacidad de escoger una o varias alternativas en función de sus propios intereses y necesidades. Un nivel que tiende hacia formas de concertación, control de la administración, cogestión y autogestión ciudadana, al darse una participación directa en la gestión de políticas y/o en el diseño de las mismas. 


\section{Políticas de participación ciudadana en el ámbito local: referentes internacionales y nacionales}

En el ámbito supranacional, las reflexiones, recomendaciones y declaraciones en torno a la participación de los ciudadanos en la vida de sus entidades locales tienen como referente importante las propuestas realizadas por la Unión Europea y la Ocde. Ambos organismos promueven acciones orientadas a fomentar la participación ciudadana en el ámbito local, compartiendo el mismo diagnóstico: la falta de interés por la política y las dificultades de implicar al ciudadano en lo público a través de los mecanismos tradicionales de participación.

Tanto la Unión Europea como la Ocde platean propuestas concretas, a través de recomendaciones, así como principios y medidas para estimular la participación de los ciudadanos. Respecto a la Unión Europea, uno de los primeros actos públicos y normativos importantes referentes a estas cuestiones fue la Recomendación $\mathrm{n}^{\mathrm{o}} \mathrm{R}$ (81) 18 del Comité de Ministros a los Estados miembros, referente a la participación en el nivel municipal. Las líneas directrices de ésta marcaron profundamente el marco estratégico de la participación en la vida local, entre otros aspectos en la necesidad de: intensificar la comunicación entre ciudadanía y representantes; mejorar las posibilidades de participación; especialmente de ciudadanos con mayores dificultades en participar activamente; incrementar la influencia de la ciudadanía en la planificación municipal, en las decisiones estratégicas para la entidad local y en sus condiciones de vida y favorecer la participación de los residentes extranjeros. Esta Recomendación es reemplazada por la Rec. (2001) 19 aprobada por el Comité de Ministros el 6 de diciembre de 2001, sobre la participación de los ciudadanos en la vida pública en el nivel local. Esta tiene por finalidad proponer a los decisores políticos un conjunto coherente y moderno de principios y líneas directivas, como base común de la acción de los Estados miembros del Consejo de Europa en este dominio esencial para la democracia. Entre sus consideraciones la citada recomendación señala expresamente que es en el nivel local donde el derecho democrático “[...] puede ejercerse más directamente y que conviene, pues, actuar para implicar a los ciudadanos de forma más directa en la gestión de los asuntos locales, salvaguardando siempre la eficacia y la eficiencia de la gestión" (Consejo de Europa, 2001). En términos similares se pronuncian instituciones como el Observatorio Internacional sobre la Democracia Participativa o la Federación Española de Municipios y Provincias, entre muchas otras.

El Consejo de Europa (2001) considera que la falta de interés por la política y las dificultades para implicar a los ciudadanos en lo público no im- 
plica un declive de la participación de los ciudadanos en la política local, sino un cambio en los modos de implicación que se expresa en la preferencia por formas directas, informales, flexibles y puntuales de participación. De ahí que recomiende a los gobiernos que elaboren una verdadera política de promoción de la participación de los ciudadanos en la vida local, con el establecimiento de medidas normativas concretas y de acciones que tengan en cuanta la importancia de la sociedad civil y las estructuras asociativas. En el ámbito local, plantea la necesidad de adaptar los procesos de toma de decisiones, utilizando mecanismos de participación tanto indirecta como directa y asociativa. Por otra parte, y atendiendo a las oportunidades y desafíos que plantea la actual sociedad de la información, el Consejo de Europa viene impulsando recomendaciones vinculadas a la "e-democracia", ante las oportunidades que esta ofrece para fortalecer la democracia, las instituciones y los procesos democráticos y vincular las T.I.C.s a la participación y la renovación del compromiso de los ciudadanos en la democracia (Rec. 2009 sobre "e-democracy" www.coe. int/democracy). ${ }^{4}$

Por su parte, la OCDE (2001) plantea, además, la preocupación por la capacidad de las entidades locales para asumir el papel que se les asigna en el contexto de la sociedad global. Plantea tres niveles de implicación de los ciudadanos en la política local: la información, la consulta y la participación activa. Según ésta institución, los tres niveles se configuran como pilares de todo buen gobierno, al permitir a los poderes públicos obtener nuevas fuentes de información y recursos para la toma de decisiones, y contribuyen a desarrollar la confianza del público en la administración, lo que incrementa la calidad de la democracia y fortalece el civismo.

Es necesario destacar que diferentes programas europeos incentivan la participación ciudadana en su elaboración, gestión y evaluación. ${ }^{5}$ Así mismo, existen organizaciones, grupos universitarios de investigación, asociaciones y redes de municipios nacionales e internaciones que difunden e intercambian experiencias participativas, asesoran a los municipios; elaboran códigos de buenas prácticas y reglamentos-tipo de participación ciudadana y promueven acciones para dar viabilidad a las buenas prácticas en las cuales se han experimentado formulas participativas.

\footnotetext{
${ }^{4}$ La e-administración e Internet pueden ser canales de transformación relacional y organizativa, aumentar la eficiencia de la gestión pública, mejorar los servicios y proveer a las acciones de gobierno de un marco más transparente.

${ }^{5}$ Tales como la Agenda Local 21, URBAN, LEADER+, EQUAL, Hábitat etc.
} 
En el ámbito nacional, la Constitución Española de 1978 realiza un mandato a los poderes públicos para promover las condiciones para que la libertad y la igualdad del individuo y de los grupos en que se integran sean reales y efectivas, remover los obstáculos que impidan o dificulten su plenitud y facilitar la participación de todos los ciudadanos en la vida política, económica, cultural y social. Todos estos principios orientan a desarrollar un sistema de servicios sociales en el que la presencia de los ciudadanos no se limite a mera recepción de prestaciones, sino que incluya, además, la colaboración de los mismos en el establecimiento de los criterios de actuación de esta materia.

En el desarrollo de estos derechos, las referencias legales básicas en el ámbito estatal son la Ley 7/1985, reguladora de las Bases de Régimen Local; el Real Decreto 2568/1986, de 28 de noviembre, por el que se aprueba el Reglamento de Organización, Funcionamiento y Régimen Jurídico de las Entidades Locales y la Ley 57/2003, de 16 de diciembre, de medidas para la Modernización del Gobierno Local. Esta última, constituye, a los efectos del presente artículo, un intento de desarrollar el tratamiento que se realiza en torno a la participación ciudadana en el ámbito local. Con esta nueva normativa, el Estado español trata de incorporarse a las tendencias europeas que proponen reforzar las posibilidades de participación y de incidencia de los ciudadanos en el gobierno local para evitar o corregir el alejamiento de éstos de la vida pública. Se destaca la necesidad de incrementar la participación y la implicación de los ciudadanos en la vida local, por lo que se establecen unos estándares mínimos y concretos que permitan hacer efectiva esa participación. Concretamente, e inspirándose en la Recomendación (2001) 19 del Consejo de Europa, mencionada anteriormente, esta nueva Ley pretende mejorar los mecanismos de participación. Entre otros, establecer reglamentos orgánicos en materia de participación ciudadana que determinen y regulen los procedimientos y mecanismos participativos, impulsar la creación de nuevos mecanismos de participación ciudadana, la combinación de éstos y la aplicación de procedimientos más ágiles con la incorporación de las nuevas tecnologías al servicio de la participación y la comunicación. La ley contempla, además, tres novedades, concretamente la creación de distritos, del Consejo Social de la Ciudad y de una Comisión Especial de Sugerencias y Reclamaciones.

Finalmente la Federación Española de Municipios y Provincias ha venido desempeñando un papel fundamental en la defensa de los intereses del municipalismo español, como en su esfuerzo de coordinación y cooperación con las otras administraciones públicas del Estado. En éste sentido, cabe resaltar la Carta de Derechos Ciudadanos y entre sus contenidos el Reglamento-Tipo de Participación Ciudadana, aprobada en 2006 y canalizada a través 
de la Agenda Local de la Participación (Alarpar), que contempla 45 líneas de actuación y recoge las tareas a desarrollar para su implementación.

\section{La participación en la reforma de las Leyes autonómicas de servicios sociales en España}

La participación ciudadana activa en los servicios sociales es un elemento esencial en la transversalidad y corresponsabilidad de las políticas sociales, facilita una orientación preventiva y educativa - no burocrática - de los servicios sociales y un modelo de gestión e intervención que posiciona de forma diferente a profesionales, instituciones, ciudadanos y entidades sociales ante los nuevos contextos. Supone concebir la participación como oportunidad y no como riesgo, dado que la inevitable incertidumbre de escuchar, dar voz y salida a las necesidades y aspiraciones ciudadanas nos hace libres. El gobierno local es un escenario privilegiado para la puesta en marcha de experiencias de participación ciudadana por su cercanía, accesibilidad, conexión y posibilidad de incluir la pluralidad y heterogeneidad de actores sociales en los asuntos públicos cotidianos. Pero ello requiere crear condiciones y contextos de participación/implicación ciudadana que generen oportunidades reales y favorables para la deliberación y construcción colectiva de las políticas sociales a partir de la conformación de preferencias sólidas e informadas entre la ciudadanía en el complejo universo relacional local.

El conjunto de leyes autonómicas de servicios sociales contempla, aunque a diferentes niveles, el principio de "participación ciudadana" o "cívica", mediante la creación de los cauces y las condiciones para impulsar la participación de la ciudadanía en la gestión del Sistema Público de Servicios Sociales, así como en la planificación, seguimiento, control y evaluación de los centros, planes y programas sociales. Las leyes más recientes identifican la promoción de la participación como finalidad (Ley 2/2007 de Cantabria, Ley 12/2008 del País Vasco), objetivo (Ley 15/2006 de Navarra, Ley 12/2007 de Cataluña, Ley 13/2008 de Galicia, Ley 5/2009 de Aragón, Ley 4/2009 de Islas Baleares), prestación (Ley 1/2003 de Asturias) de las políticas de servicios sociales, competencia municipal y/o función específica de los servicios sociales de base.

Así mismo, contempla la creación de mecanismos que canalicen los derechos y deberes de las personas usuarias de centros, servicios y programas, bien directamente de manera individual o a través de entidades sociales representativas (no lucrativas, iniciativa y voluntariado). Ambos ejercicios de ciudadanía se plasman, especialmente en las leyes promulgadas a partir de 
2005 que refuerzan la protección de los usuarios, con un principio de garantía de la participación y una descripción detallada de derechos y deberes, entre los que se identifica la participación de las personas como agentes de su propio cambio y de los grupos y entidades de la sociedad civil en el funcionamiento del Sistema de Servicios Sociales.

Con el fin de garantizar la participación en la planificación y gestión del Sistema Público de Servicios Sociales, contemplan la creación de órganos asesores y consultivos de participación ciudadana y asociativa: Consejos Locales de Bienestar Social, Territoriales, Locales y Sectoriales de Servicios Sociales, según los casos.

Respecto a la participación de las personas usuarias, las leyes de "segunda" y "tercera" generación les atribuyen, al menos formalmente, un papel más activo, concretamente participar en todas las decisiones que les afecten de forma directa o indirecta, individual o colectiva. Todas las entidades y centros de servicios sociales deberán contar con procedimientos de participación democrática de las personas usuarias, o de sus representantes legales, de acuerdo con lo que se determine reglamentariamente; a tal efecto establecen como mecanismo el consejo de usuarios.

Resulta de interés destacar los derechos y deberes de los usuarios/destinatarios que incorporan las recientes leyes, concibiendo los servicios sociales como derecho subjetivo y con una clara sintonía/influencia/integración de la Ley de Promoción de la Autonomía Personal y Atención a la Dependencia. Entre los derechos destacar: acceder en condiciones de igualdad, dignidad y privacidad; confidencialidad; autonomía; dar o denegar consentimiento respecto de una intervención; dar instrucciones previas para situaciones futuras de incapacidad respecto a asistencia o cuidados que se le puedan procurar y derecho a la autotutela; renunciar a prestaciones y servicios concedidos; disponer de información suficiente, veraz y fácilmente comprensible sobre las intervenciones propuestas, servicios sociales disponibles y requisitos necesarios para su acceso; disponer de un profesional de referencia y una evaluación o diagnóstico de sus necesidades; participar, individual o colectivamente, en las decisiones que les afecten y en el funcionamiento de los servicios y acceder a cauces de información, sugerencia y queja que permitan el ejercicio efectivo y la defensa de sus derechos; a escoger libremente; ser atendidas en función de su propia preferencia; a la calidad de las prestaciones y servicios etc.

Respecto a los deberes: cumplir normas, requisitos, condiciones y procedimientos establecidos, respetar el plan de atención personalizada y las orientaciones; facilitar la información necesaria y veraz; destinar las prestaciones a la finalidad para las que hubieran sido concedidas etc. 
Los servicios sociales municipales deben facilitar el acceso y la participación de los ciudadanos no como usuarios o meros beneficiarios de actividades, sino en la elaboración, diseño, seguimiento y evaluación de programas, en la gestión de los Centros de Servicios Sociales, en definitiva, en pasar de una participación "interesada", de meros "espectadores" a una participación "sustantiva". Desde esta orientación participativa será posible acercar los discursos de los actores implicados para articular las respuestas necesarias a los complejos escenarios a los que se enfrentan los servicios sociales municipales.

\section{Dimensiones y mecanismos de participación en las políticas sociales municipales}

A pesar de la heterogeneidad de mecanismos de participación existentes, unido a su incesante evolución e innovación, es posible sistematizar este complejo escenario de experiencias atendiendo a las diferentes tipologías de clasificación: nivel de institucionalización; formalización y estabilidad; nivel jurisdiccional al que afecta la decisión; nivel de participación ofrecido; fuente de la iniciativa; carácter de sus competencias (territorial o sector); fase del proceso de actuación política sobre el que se proyecta; interés u objetivos que se tengan o base de la participación. Centrándonos en la última por su utilidad comparativa y de análisis posterior, podemos distinguir:

a) Asociativa, se canaliza a través de grupos organizados de ciudadanos. Son mecanismos muy extendidos en los municipios españoles, especialmente urbanos, en el diagnóstico y la formación de la agenda local, así como en la gestión de equipamientos, servicios y programas. Se articula, fundamentalmente, a través de: consejos municipales de participación ciudadana; consejos territoriales o sectoriales; comités de usuarios de los servicios y agrupaciones de desarrollo local.

b) Personal directa, son espacios de participación para los ciudadanos individualmente considerados. En ocasiones se establecen criterios representativos en la selección de los participantes, mientras que otros es aleatorio. En éste ámbito podríamos citar experiencias tales como: presupuestos participativos; jurados ciudadanos o núcleos de intervención participativa; asambleas de ciudadanos; reuniones vecinales, comunales o de servicios; referéndum o consulta popular; encuestas de satisfacción; encuestas deliberativas; grupos de discusión etc. 
c) Mixta, combinan los dos anteriores: planes estratégicos territoriales; agendas21; consejos, foros o asambleas territoriales, sectoriales o de servicios; plataformas ciudadanas; talleres de prospectiva de territorios y servicios.

Del análisis de las investigaciones sobre participación en el ámbito local en el ámbito europeo y nacional, ${ }^{6}$ podemos constatar dos fenómenos: por una parte, la descentralización del Estado de Bienestar a partir de mediados de los ochenta ha provocado que los gobiernos municipales, especialmente urbanos, hayan tenido que buscar interlocutores sociales - tercer sector/sistema - en relación con sus nuevas competencias; y, por otra, que los gobiernos municipales emplean estrategias adaptativas en relación con las rasgos estables y dinámicos de su estructura política en su trayectoria histórica (Navarro, 2002:40-41). De ahí que la oferta de oportunidades de participación política que impulsan, inicialmente, los ayuntamientos, fundamentalmente urbanos, se base en el modelo tradicional y típicamente asociativo (consejos territoriales y sectoriales), aunque cada vez más se vienen poniendo en marcha mecanismos de participación directa - modelo de orientación ciudadanista - bien de información y/o consulta (encuestas, defensor del ciudadano, referéndum, Internet) y/o deliberativa, que suponen un proceso de discusión pública en torno a decisiones y/o iniciativas concretas (presupuestos participativos, consejos ciudadanos) (Navarro, 2008).

Considerado el nivel de formalización y objeto principal adoptado por los mecanismos de participación mayoritarios en las políticas de servicios sociales, podemos distinguir: estructuras de participación de base asociativa o consejos territoriales y locales (consultivos y/o asesores); organismos públicos autónomos desconcentrados o institutos; consejos de usuarios y mecanismos no formales (dimensión informal del ejercicio de facilitación). Los mecanismos de participación ciudadana mayoritarios en las políticas de servicios sociales municipales podemos caracterizarlos de la siguiente forma:

a) Estructuras de participación de base asociativa o consejos. Se caracterizan por ser consultivos, de ámbito territorial y/o sectorial, de carácter extensivo y facilitadoras de información, asesoramiento y captación de propuestas.

b) Organismos públicos autónomos desconcentrados o Institutos. Disponen de personalidad jurídica propia, delegación de competencias en servicios so-

\footnotetext{
${ }^{6}$ Entre otros: Sarasa y Obrador, 1999:117-147; Alguacil, 2000:157-177; Font, 2001; Navarro, 2002; Del Pino y Colino, 2003; Brugué y Vallés, 2005; Gadea, 2005; Gutiérrez, 2005; Montero, Font y Torcal, 2006; Espadas, 2007:155-176; Alguacil, 2008; Inap, 2008; Navarro, 2008; Pindado, 2008; Pastor, 2009 etc.
} 
ciales generales y/o sectoriales y con cierta autonomía en la toma de decisiones y la gestión económico-administrativa y técnica del área. De esta forma, combinan: adopción de decisiones, ejecución/gestión de acuerdos y participación.

c) Mecanismos no formales de participación de base asociativa (dimensión informal del ejercicio de facilitación), a través de reuniones periódicas protagonizadas por técnicos de servicios sociales con organizaciones del territorio y técnicos de otros sistemas. La iniciativa, convocatoria, agenda y organización se realiza a través de los directores/coordinadores de los Centros de Servicios Sociales, en ocasiones sin respaldo sobre los asuntos tratados y acuerdos alcanzados en ellas. Entre sus fines: ofrecer información, detectar necesidades; solicitar participación, promover la cooperación interasociativa y generar redes y protocolos de colaboración técnica interinstitucional.

\section{Rendimiento democrático de los órganos de participación institucionalizados (análisis de caso región de Murcia, España)}

El análisis de la regulación normativa de los consejos constata que ésta otorga a la administración una enorme y flexible capacidad de control sobre la agenda y los procesos participativos. Los estatutos y reglamentos definen y determinan los niveles y asuntos sobre los qué es posible participar, limitando de ésta forma el marco y posibilidades reales de participación efectiva sobre los asuntos sociales públicos, a la vez que permiten al responsable político/ técnico introducir en la agenda de las sesiones aquellas cuestiones que a éste le interesan. De esta forma, los participantes se convierten en "consumidores-invitados" pero no protagonistas, pueden hacer valer su voz, pero filtrada en atención a su encaje en la agenda y estrategia de la acción política. Un diseño que introduce evidentes asimetrías en las oportunidades de participar y refuerza la dependencia de las organizaciones sociales respecto de la administración. En definitiva, un modelo centrado en lo institucional y en procedimientos previstos y controlados por y para la administración.

La composición y representatividad de los consejos tienen una base de participación, fundamentalmente, asociativa; con un claro protagonismo de las federaciones, fundaciones y asociaciones frente a los ciudadanos de forma individual, plataformas y entidades minoritarias; a la vez que se privilegia la representación sectorial a la territorial. Estas preferencias por las entidades 
sociales, fundamentalmente sectoriales (carácter especializado), como protagonistas en la participación, es una pauta interaccional muy habitual en las políticas y experiencias participativas.

Los gobiernos municipales distribuyen de modo diverso las oportunidades de participación en la trama asociativa local. El cierre de oportunidades de participación parece deberse más a la forma en que se distribuyen tales oportunidades que a la ausencia de interés por parte de las asociaciones, ya que, al existir invitación, las asociaciones suelen participar. ${ }^{7}$ De esta forma, los gobiernos locales se reservan la oferta de oportunidades de participación, diseñan y adaptan la red de actores incluidos (y por tanto excluidos), en una coalición que no ponga en peligro las orientaciones y acciones previstas.

La fragmentada y atomizada realidad asociativa dificulta procesos de representación y participación plural, especialmente en los municipios de mayor tamaño donde el tejido social es muy numeroso y diverso. Ciertos grupos tienden a acaparar la representación social en múltiples foros participativos y se observan contextos asimétricos de capacidad y oportunidad de acceder a los asuntos políticos. La desigualdad, por una parte, en el acceso a los espacios y oportunidades de participación de las organizaciones y, por otra, la capacidad y habilidad de los participantes en formular opiniones políticas, ${ }^{8}$ determina la asimetría en la representación de los diferentes colectivos y problemáticas sujetos y objeto de la acción de los servicios sociales.

Los objetivos de los consejos son de carácter consultivo, no vinculantes para las autoridades (dar y recoger información) y, por tanto, basados en un concepto restringido de participación, entendida en términos de información, consulta y colaboración. Se constata la existencia de una brecha entre los objetivos formales y reales que se alcanzan en la práctica, así como en la distinta percepción que los actores implicados tienen acerca de los objetivos que tienen y que debieran cumplir (expectativas). De un lado, las entidades sociales implicadas los perciben como un instrumento para mantenerse informadas sobre los asuntos sociales municipales; intercambiar puntos de vista; informar

\footnotetext{
${ }^{7}$ Tal y como se constata en el estudio realizado por NAVARRO, Clemente Y JUARISTA, P., "Funciones, actividades y facilitación pública de las asociaciones", en J. R. Montero, J. Font y M. Torcal (ed.), Ciudadanos, asociaciones y participación en España. 2006. Madrid: C.I.S. p. 231-232.

${ }^{8}$ Una desigual capacidad y habilidad de los participantes para formular opiniones políticas debido al insuficiente capital cultural y/o autonomía organizativa; dado que "no todos [...] poseen en el mismo grado los instrumentos, especialmente el capital cultural, que son necesarios para producir una opinión personal, en el doble sentido de autónoma y conforme a los intereses vinculados a una posición particular", como señala Bourdieu, 2005:75.
} 
a la administración de las acciones que realizan; disponer de un espacio de encuentro y colaboración entre entidades sociales que les permite conocer la existencia y el trabajo que cada una realiza; coordinar servicios y actuaciones y trasladar necesidades y reivindicaciones de colectivos. Esta comunicación ha mejorado, en algunos casos, la derivación de casos y el desarrollo de acciones concretas entre los Centros de Servicios Sociales y las organizaciones sociales. Estas valoran muy favorablemente estos mecanismos especialmente por la posibilidad de diálogo e intercambio de información con los profesionales de los servicios sociales. Para los técnicos supone un instrumento para detectar necesidades sociales, agilizar procesos y, en ocasiones, mejorar la coordinación, de las cuestiones cotidianas del área (seguimiento de casos, altas y bajas en prestaciones económicas y servicios, información de proyectos y resultados).

La función práctica de los consejos es dar cuenta y recibir información acerca de los proyectos realizados o a implantar y de los casos que requieren de coordinación de recursos. El debate y la deliberación de los asuntos para la elaboración de propuestas, aunque éstas no sean vinculantes, son casi inexistentes. Pero ello no debe imputarse directamente a los representantes del gobierno local, sino a la misma dinámica comunicativa que se genera en el nivel de participación que ofrecen estos mecanismos y por la función adaptativa de las propias entidades sociales. Uno de los aspectos más visibles e importantes de la participación analizada es su competencia a la hora de determinar las formas de hacer, los aspectos metodológicos de las sesiones, las cuestiones y la forma en como éstas se abordan no facilitan la consulta, deliberación y elaboración de propuestas. El proceso participativo es percibido y considerado un trámite más administrativo que sustantivo.

Los asuntos tratados se relacionan con la presentación e información de servicios, proyectos actuaciones, presupuesto, normativas reguladoras de prestaciones y ayudas y casos.

Se observa que la ciudadanía en general y las organizaciones sociales no participantes desconocen la existencia y/o funcionamiento de los consejos, dado que el proceso de formalización y funcionamiento no ha estado acompañado de actuaciones, previas y posteriores, suficientes de información, difusión, consulta, propuesta y debate. Esta insuficiente transparencia retroalimenta/contribuye a la arbitrariedad en la selección de actores y funcionamiento de los mecanismos, reproduciendo sesgos en la participación.

Por otra parte, los acuerdos entre organizaciones y administración se adoptan, habitualmente, de forma bilateral y en espacios diferentes a los consejos. El resultado es que las decisiones no se adoptan en esferas colegiadas y participativas entre todas las organizaciones, sino que son discutidas y acor- 
dadas en ámbitos "privados". El silencio de los acuerdos adoptados al margen, en el actual contexto pluralista, condiciona la acción y los discursos de profesionales, organizaciones y políticos, tanto en el presente como en el futuro, influyendo negativamente en las relaciones interasociativas y en los procesos de participación de acción colectiva.

\section{Conclusiones y oportunidades para ejercer ciudadanía}

Los resultados del estudio empírico informan que el modelo de consejos e institutos no ha cumplido sus objetivos cuantitativos de cobertura ni cualitativos en referencia a la calidad de participación ofrecida y percibida. Así mismo, se observa una escasa implicación/participación activa de los miembros, entendida ésta como la formulación razonada de propuestas e iniciativas concretas sobre los asuntos sociales públicos que en estos se tratan.

La política de participación ciudadana a través de los consejos e institutos no obedece a un proyecto de profundización democrática de la Política de Servicios Sociales municipales, en el sentido de una transferencia de poder a la ciudadanía en la gestión de los asuntos sociales públicos, sino a una opción de carácter meramente distributivo, mediante la intensificación de la estrategia relacional que permite aumentar la eficacia de la acción del gobierno y profesionales. Mecanismos que fortalecen los aspectos más formales, representativos y simbólicos de la participación en detrimento de otros como la deliberación, conflicto, equidad, cambio social y adopción colegiada de decisiones y que sugieren fuerza real a la acción comunicativa de los actores sociales.

Subyace la consideración del papel de los ciudadanos como meros usuarios, consumidores, clientes, receptores o destinatarios de servicios y no como ciudadanos, como actores políticos capaces de intervenir e influir en la gestión de los asuntos y las políticas públicas (potenciación o capacitación - "empowerment"). La participación se limita a elegir sobre determinadas opciones, criterios y políticas previamente definidas y delimitadas. No existe posibilidad real de definir necesidades ni las formas de satisfacerlas y, por tanto, se constata una escasa capacidad de intervenir de manera efectiva en las decisiones de la administración. De esta forma, como señala Pindado (2008), se pierde la oportunidad de aprovechar la energía ciudadana para ayudar a transformar la realidad y alcanzar mayores cotas de bienestar y felicidad. Sin embargo, existe coincidencia entre los informantes al señalar una escasa participación activa de los miembros de los consejos, entendida como la formulación razonada de 
propuestas sobre los asuntos que se debaten. Esta aparente contradicción es motivada por el funcionamiento y organización de los mecanismos (cómo) — condiciones comunicativas como señala Habermas - , pero también por las diferentes y divergentes expectativas de los actores respecto de la participación en los mismos.

En coincidencia con las conclusiones de estudios sobre la democracia asociativa, la política municipal de participación en los servicios sociales municipales conforma un modelo democrático que puede denominarse de "democracia de acceso", pues queda fuera de la incorporación de la ciudadanía a la formulación de las políticas, sin favorecer la posibilidad de realizar un ejercicio de poder efectivo, no ya en la activación de la agenda, sino en la discusión en torno a los problemas que ésta debe o no debe incluir. Se produce una división que "separa el conjunto de las cosas importantes, por un lado, de las cosas accesorias, por otro" (Gutiérrez, 2005:180). Mientras, el discurso de la participación institucional se refiere a ésta como deliberación y discusión de los asuntos públicos, la realidad demuestra que los consejos se ocupan de los aspectos secundarios. La participación que promueven las administraciones locales en materia de servicios sociales es formal, las formas pasan a ser el mejor exponente de los derechos sin perder cierto carácter instrumental en el sentido en que sirve para legitimar las políticas de las instituciones y, en ocasiones, de los profesionales de los centros de servicios sociales.

Del análisis de las experiencias no se desprende un aprendizaje democrático de los actores participantes ni se ha logrado afianzar posturas de acción política colectiva. Por el contrario, se observa escepticismo, inmovilismo, desconfianza y cansancio y una cierta inercia institucionalizada sobre las formas y posibilidades del participar. Consideran escasas las posibilidades de desarrollo de un modelo de participación real más allá del modelo institucional y formal instaurado. Albergan esperanzas de cambios condicionados a cambios en el sistema político, lo que nos vuelve a confirmar que los mecanismos de participación se encuentran sujetos al oportunismo y arbitrariedad política.

Los consejos e institutos son socializantes, educativos, favorecen la conciencia y corresponsabilidad social, generan actitudes y comportamientos solidarios. Pero estos valores requieren de la percepción de transparencia en la gestión de la participación y en su consideración sustantiva. Los actores dejan de asumir estos principios cooperadores cuando se sienten "utilizados" para dar cumplimiento a una norma o servir de "pantalla pública" con la que decir que se da participación a los ciudadanos. De esta forma su participación deja de ser cooperadora y solidaria y se convierte, aunque legítimamente, en "interesada" en los asuntos particulares que interesan al colectivo o asociación 
a la que representan, pasando de ser sustantiva a "egocéntrica", y, por tanto, inhabilitada para generar procesos colectivos.

Los consejos e institutos favorecen procesos de interacción entre los actores pero no garantizan por sí mismos, y de forma automática, una democratización en la construcción de las políticas sociales en el ámbito local, ni viceversa. No favorecen la posibilidad de realizar un ejercicio de poder efectivo, no ya en la activación de la agenda, sino en la discusión en torno a los problemas que ésta debe o no debe incluir. De ahí, que más allá de su regulación precisa de un diseño, organización y funcionamiento que posibilite condiciones y oportunidades reales para acceder e influir en los procesos de toma de decisiones respecto de las políticas sociales. La eficacia en la gestión de los asuntos sociales no debe dejar en un segundo plano la concepción de estos mecanismos como instrumentos de democratización.

\section{Referencias}

ALGUACIL, Julio. Ciudad, ciudadanía y democracia urbana. Revista de Documentación Social Monográfica: ciudades habitables y solidarias, n. 119, p. 157-177, 2000.

. Espacio público y espacio político. La ciudad como el lugar para las estrategias de participación. Revista Académica de la Universidad Bolivariana, n. 20, 2008.

ARENDT, Hannab. ¿Qué es la política?. Barcelona: Paidós, 1997.

ARNSTEIN, Sherry R. A ladder of citizen participation. Journal of the American Institute of Planners, v. 35, n. 4, p. 216-224, 1969.

ARRIBA, Ana; CALZADA, Inés; DEL PINO, Eloisa. Las actitudes de los españoles hacia el Estado de Bienestar (1985-2005). Madrid: CIS, 2006.

BECK, Ulrico. La sociedad del riesgo mundial: en busca de la seguridad perdida. Barcelona: Paidós, 2008.

BOURDIEU, Pierre. El misterio del ministerio. De las voluntades particulares a la voluntad general. En: WACQUANT, L. (Coord.). El misterio del ministerio. Pierre Bourdieu y la política democrática. Barcelona: Gedisa, 2005.

BRUGUÉ, Quim; VALLÉS, Joseph M. Nuevos ayuntamientos, concejales diferentes: del gobierno de las instituciones al gobierno de las redes. Madrid: F.E.M.P., 2005.

CONSEJO DE EUROPA. Recomendación no (2001) 19 del Comité de Ministros a los Estados Miembros aprobada por el Comité de Ministros en 6 de diciembre de 2001. Disponible en: <www.coe.int/>. 
CUNILL, N. Participación ciudadana: Dilemas y perspectivas para la democratización de los estados latinoamericanos. Caracas: Clad, 1991.

DE LA TORRE, Isabel. Tercer sector y participación ciudadana en España. Madrid: C.I.S., 2005. (Colección: Opiniones y Actitudes, n. 51)

DEL PINO, Eloisa; COLINO, Cesar. Los ciudadanos y el Estado: las actitudes de los españoles hacia las administraciones y las políticas públicas. Madrid: I.N.A.P., 2003.

ESPADAS, Ma. Angeles. Tercer sector y reestructuración del bienestar: aspectos cuantitativos de la gestión de los servicios sociales en Andalucía. Revista Documentación Social, Madrid, n. 146, p. 155-176, 2007.

F.E.M.P. Encuesta sobre la participación ciudadana en los ayuntamientos. Madrid, 2002.

FONT, Joan. Ciudadanos y decisiones políticas. Barcelona: Ariel, 2001.

; BLANCO, Ismael. Nuevos mecanismos participativos y democracia participativa. Ponencia en Jornadas sobre democracia participativa, U.P.V., 2003.

; SAN MARTÍN, Joseph; SCHNEIDER, Cecili. Asociaciones y democracia: contribuciones y causalidades. En: MONTERO, José Ramón; FONT, Joan; TORCAL, Mariano (Ed.). Ciudadanos, asociaciones y participación en España. Madrid: C.I.S., 2006. p. 241-259.

FOX, Charles; MILLER'S, Hugo. Postmodern public administration. Londres: Sage, 1995.

FRESNO, J.M. (Coord.). Políticas de inclusión activa en el plano local. Madrid: Fundación Luis Vives, 2009.

GADEA, Elena. Las políticas de participación ciudadana: nuevas formas de relación entre la administración pública y la ciudadanía. Universidad de Valencia, 2005.

GIDDENS, Anthony. Más allá de la izquierda y la derecha, el futuro de las políticas radicales. Madrid: Cátedra, 1998.

GUTIÉRREZ SASTRE, Marta. La participación en los servicios públicos de bienestar: Un análisis del poder de los usuarios en el ámbito de la salud, la educación y los servicios sociales. Madrid: C.E.S., 2005. (Colección Estudios, n. 177)

HELD, D. La democracia y el orden global: del Estado moderno al gobierno cosmopolita. Barcelona. Paidós, 1997.

HIRSCHMAN, A. Salida, voz y lealtad. México: Fondo de Cultura Económica, 1997. 
I.N.A.P. Evaluación de la participación pública en la elaboración de las políticas públicas. Madrid: Ministerio de Administraciones Públicas, 2008.

LÖFFLER, E. Experiencias internacionales de participación ciudadana en Europa. In: CONGRESO INTERNACIONAL DE DEMOCRACIA PARTICIPATIVA, 1ํ. 2005.

MONTERO, José Ramón; FONT, Joan; TORCAL, Mariano (Ed.). Ciudadanos, asociaciones y participación en España. Madrid: C.I.S., 2006.

MORALES, Laura. ¿Existe una crisis participativa? La evolución de la participación política y el asociacionismo en España. Revista Española de Ciencia Política, Madrid, n. 13, p. 51-87, 2005.

NAVARRO YÁÑEZ, Clemente, J. Democracia asociativa y oportunismo político: la política pública de participación ciudadana en los municipios españoles (19791993). Valencia: Tirant lo Blanch, 2002.

; JUARISTA, P. Funciones, actividades y facilitación pública de las asociaciones. En: MONTERO, J.R.; FONT, J.; TORCAL, M. (Ed.). Ciudadanos, asociaciones y participación en España. Madrid: C.I.S., 2006. p. 223-240.

. Participación local. Estudio C.I.S., Madrid, n. 2.661, 2008.

O.C.D.E. Implicar a los ciudadanos en la elaboración de políticas: información, consulta y participación pública. En: PUMA. Nota de políticas públicas sobre gestión pública, 10. Paris, 2001. Disponível em: <www.oecd.org>.

PASTOR SELLER, Enrique. Trabajo social comunitario. Murcia: Diego Marín, 2006.

. La participación ciudadana en los servicios sociales municipales de la región de Murcia. Murcia. Murcia: Universidad de Murcia, 2008a.

. Ciudadanía, democracia y política social municipal. Revista de Ciencias Sociales Sistema, Madrid, n. 207, p. 109-127, 2008b.

. Participación ciudadana y gestión de políticas sociales municipales. Murcia: Editum, 2009.

PINDADO SÁNCHEZ, Fernando. La participación ciudadana en el ámbito local. Madrid: Femp, 2008.

PUTNAM, R.D. El declive del capital social: un estudio internacional sobre las sociedades y el sentido comunitario. Barcelona: Galaxia Gutemberg-Circulo e Lectores, 2003.

PRUITT, Bettye; THOMAS, Philip. Diálogo democrático. Pnud, 2008. 
RODRÍGUEZ CABRERO, Gregorio; CODORNIZ, M. Las entidades voluntarias en España, institucionalización, estructura económica y desarrollo asociativo. Madrid: Ministerio de Asuntos Sociales, 2000.

et al. Actores sociales y reformas del bienestar. Madrid: Consejo Superior de Investigaciones Científicas, 2005.

RODRÍGUEZ HERRERO, Gorka; AJANGIZ, Rafael. Descentralización municipal y participación ciudadana: evaluación de una experiencia consolidada. Revista Española de Ciencia Política, Madrid, n. 17, p. 181-197, 2007.

RUIZ OLABUENAGA, J.I. (Dir). El sector no lucrativo en España: una visión reciente. Bilbao: Fundación B.B.V.A., 2006.

SALAMON, Lester M. et al. La sociedad civil global: las dimensiones del sector no lucrativo - proyecto de estudio comparativo del sector no lucrativo. Bilbao: Fundación B.B.V.A., 2001.

SARASA, Sebastián; OBRADOR, G. El papel de la sociedad civil en los servicios sociales. En: SUBIRATS, J. (Ed.). ¿Existe sociedad civil en España? Madrid: Función Encuentro, 1999. p. 117-147.

SUBIRATS, Joan. Los servicios sociales de atención primaria ante el cambio social. Madrid: Ministerio de Trabajo y Asuntos Sociales, 2007.

VVAA. Informe España 2005: una interpretación de su realidad social. Madrid: Cecs, 2005.

WARREN, M.E. Democracy and association. Princeton: Princeton University Presss, 2001. 